\title{
Resonant Ultrarelativistic Electron-Positron Pair Production by High-Energy Electrons in the Field of an X-ray Pulsar
}

\author{
Georgii K. Sizykh *, Sergei P. Roshchupkin (1) and Victor V. Dubov \\ Department of Theoretical Physics, Peter the Great St. Petersburg Polytechnic University, Polytechnicheskaya 29, \\ 195251 Saint Petersburg, Russia; roshchupkin_s@spbstu.ru (S.P.R.); dubov@spbstu.ru (V.V.D.) \\ * Correspondence: GeorgiiSizykh@yandex.ru
}

Received: 14 July 2020; Accepted: 19 August 2020; Published: 22 August 2020

check for updates

\begin{abstract}
The process of resonant high-energy electron-positron pair production by an ultrarelativistic electron colliding with the field of an X-ray pulsar is theoretically investigated. Resonant kinematics of the process is studied in detail. Under the resonance condition, the intermediate virtual photon in the X-ray pulsar field becomes a real particle. As a result, the initial process of the second order in the fine structure constant effectively reduces into two successive processes of the first order: X-ray-stimulated Compton effect and X-ray-stimulated Breit-Wheeler process. For a high-energy initial electron all the final ultrarelativistic particles propagate in a narrow cone along the direction of the initial electron momentum. The presence of threshold energy for the initial electron which is of order of $100 \mathrm{MeV}$ for $1-\mathrm{KeV}$-frequency field is shown. At the same time, the energy spectrum of the final particles (two electrons and a positron) highly depends on their exit angles and on the initial electron energy. This result significantly distinguishes the resonant process from the non-resonant one. It is shown that the resonant differential probability significantly exceeds the non-resonant one.
\end{abstract}

Keywords: external field QED; X-ray pulsars; high-energy particles; resonance; trident pair production; positrons; positron abundance

PACS: 95.30.Cq; 97.10.Ld; 97.80.Jp

\section{Introduction}

Resonant nonlinear effects of quantum electrodynamics (QED) can be observed in strong electromagnetic fields. To create such fields and ultrarelativistic particles special conditions are required. These conditions can be achieved near specific space objects, such as neutron stars, pulsars, magnetars or supernovae. It has been reported of anomalous high-energy positron abundance [1] in cosmic rays, which are suggested to be produced by pulsars [2]. Several external field QED effects make contribution to production of high-energy positrons. In this paper resonant ultrarelativistic electron-positron pair production by high-energy electrons in the field of the X-ray pulsar is studied. The possibility of production of positrons with energies discussed in [1] will be shown.

The first time is was Oleinik [3,4] (see also reviews [5,6]), who discovered the presence of resonances in second-order external field QED processes. The resonances are related with discrete energy spectrum of particle + external field system. Under the resonance condition intermediate photon becomes a real particle and the process' probability greatly increases. One more factor distinguishing resonant processes from the non-resonant one is existence of dependencies of final particles energies on their outgoing angles. Resonant QED processes in an external field were studied in works [7-22]. 
The process of high-energy electron-positron pair production by an ultrarelativistic electron colliding with an external field is studied in works [23-27]. In work [23] authors put special emphasis on properties of transition amplitude and study it analytically and numerically. In work [24] authors study the process in inhomogeneous plane-wave background fields, the probability of the process, including the exchange part is obtained. In works [25-27] one-step and two-step contributions to the process are studied in detail.

In contrast to the works discussed above in this paper the resonant high-energy electron-positron pair production by an ultrarelativistic electron colliding with the field of an X-ray pulsar is theoretically investigated. We consider ultrarelativistic particles which leads to the fact that all the final particles in the process propagate along the direction of the initial electron. We mostly focus on resonant kinematics of the process. We also aim to compare the differential probability of the process and the differential probability of the non-resonant one.

\section{External Field Description}

QED processes in an external electromagnetic field are described in terms of classical relativistically invariant parameters:

$$
\eta=\frac{e F \lambda}{m c^{2}}
$$

the physical meaning of which is the ratio of the work of electromagnetic field at its wavelength to an electron (positron) rest energy. Here $e$ and $m$ are the charge and the mass of an electron (positron), $\lambda$ is the reduced wavelength of the field, $c$ is the speed of light and $F$ is the strength of the field.

Fox example, for an X-ray pulsar with $\omega=1 \mathrm{KeV}$ and $F=10^{11} \mathrm{~V} / \mathrm{cm}$ parameter $\eta$ takes value of order of $10^{-2}$.

In this paper, we consider weak electromagnetic fields which are defined by the following condition:

$$
\eta \ll 1
$$

We assume the field of a pulsar to be monochromatic electromagnetic circularly polarized plane wave of the following form:

$$
A(\varphi)=\frac{F}{\omega}\left(e_{x} \cos \varphi+\delta e_{y} \sin \varphi\right), \quad \varphi=k x=\omega(t-z) .
$$

In Equation (3) $A$ and $\omega$ are the 4-potential and the frequency of the wave, $e_{x}$ and $e_{y}$ are polarization 4 -vectors and $\delta= \pm 1$.

Further we use relativistic system of units: $\hbar=c=1$.

\section{Amplitude of Ultrarelativistic Electron-Positron Pair Production}

The process of resonant electron-positron pair production by an electron in the field of an X-ray pulsar can be described in terms of two Feynman diagrams as shown in Figure 1. Double lines correspond to Volkov functions [28], the solutions of Dirac equation with monochromatic electromagnetic plane wave potential.
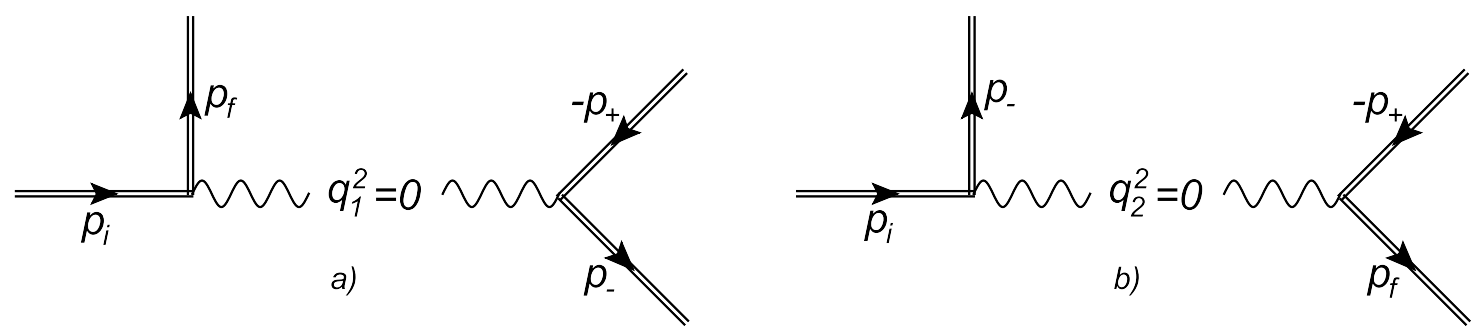

Figure 1. Feynman diagrams of resonant electron-positron pair production by an electron in the field of the X-ray pulsar. (a)—channel "a"; (b)—channel "b". Double lines correspond to Volkov functions. 
The amplitude of the process can by written by means of standard Feynman rules:

$$
S=i e^{2} \int d^{4} x_{1} d^{4} x_{2} \bar{\Psi}_{p_{-}}\left(x_{2}\right) \gamma^{\nu} \Psi_{-p_{+}}\left(x_{2}\right) D_{\mu v}\left(x_{2}-x_{1}\right) \bar{\Psi}_{p_{f}}\left(x_{1}\right) \gamma^{\mu} \Psi_{p_{i}}\left(x_{1}\right)-\left(p_{f} \longleftrightarrow p_{-}\right) .
$$

Here $p_{i, f}=\left(E_{i, f}, \boldsymbol{p}_{i, f}\right)$ is the 4-momentum of initial and final electrons, $p_{ \pm}=\left(E_{ \pm}, \boldsymbol{p}_{ \pm}\right)$is the 4-momentum of positron and electron of pair and

$$
\Psi_{p}(x)=\left[1+\frac{e}{2(k p)} \hat{k} \hat{A}\left(\varphi_{1}\right)\right] \frac{u_{p}}{\sqrt{2 E}} e^{i S_{p}(x)} .
$$

is the Volkov function of an electron (positron). $\bar{\Psi}_{p}$ denotes Dirac adjoint of $\Psi_{p}$ and $-p_{+}$stands to underline that positron Volkov function can formally be obtained from the electron one by change of the sign of 4-momentum, $u_{p}$ is free Dirac bispinor.

In Equation (5)

$$
S_{p}(x)=-p x-\int_{\varphi_{0}}^{\varphi} \frac{e}{(k p)}\left[\left(p A\left(\varphi^{\prime}\right)\right)-\frac{e}{2} A^{2}\left(\varphi^{\prime}\right)\right] d \varphi^{\prime}
$$

is the classical action of an electron in the field of a monochromatic electromagnetic plane wave [29].

After integrations in Equation (4) the amplitude of the process in case of weak (see Equation (2) circularly polarized wave takes the form of

$$
S=\sum_{l=-\infty}^{\infty} S_{l}
$$

where $S_{l}$ are partial amplitudes, corresponding to $l$ photons of the external field absorbed in the process and given by the following expression:

$$
\begin{aligned}
S_{l}= & 16 \pi^{5} e^{2} \frac{e^{-i d^{*}}}{\sqrt{E_{i} E_{f} E_{-} E_{+}}} \sum_{l_{2}=1}^{\infty} \frac{\delta^{(4)}\left(p_{i}-p_{f}-p_{+}-p_{-}+l k\right)}{\left(p_{+}+p_{-}-l_{2} k\right)^{2}} \times \\
& \times\left[\bar{u}_{p_{-}} H_{\mu ;-l_{2}} v_{-p_{+}}\right]\left[\bar{u}_{p_{f}} H_{-\left(l-l_{2}\right)}^{\prime \mu} u_{p_{i}}\right]-\left(p_{f} \longleftrightarrow p_{-}\right)
\end{aligned},
$$

where $d^{*}$ is an independent of the summation index phase.

Here

$$
\begin{array}{r}
H_{l_{2}}^{\mu}=\gamma^{\mu} L_{l_{2}}\left(\gamma_{p_{+} p_{-},} \chi_{p_{+} p_{-}}\right)+b_{-}^{\mu} L_{l_{2}-1}\left(\gamma_{p_{+} p_{-}}, \chi_{p_{+} p_{-}}\right)+b_{+}^{\mu} L_{l_{2}+1}\left(\gamma_{p_{+} p_{-},} \chi_{p_{+} p_{-}}\right) \\
H_{l_{1}}^{\prime \mu}=\gamma^{\prime \mu} L_{l_{1}}\left(\gamma_{p_{i} p_{f}}, \chi_{p_{i} p_{f}}\right)+b^{\prime \mu} L_{l_{1}-1}\left(\gamma_{p_{i} p_{f}}, \chi_{p_{i} p_{f}}\right)+b_{+}^{\prime \mu} L_{l_{1}+1}\left(\gamma_{p_{i} p_{f}}, \chi_{p_{i} p_{f}}\right)
\end{array}
$$

where

$$
b_{ \pm}^{\mu}=\eta\left[\frac{m}{4\left(k p_{+}\right)} \gamma^{\mu} \hat{k} \hat{\varepsilon}_{ \pm}-\frac{m}{4\left(k p_{-}\right)} \hat{\varepsilon}+\hat{k} \gamma^{\mu}\right], \quad b_{ \pm}^{\prime \mu}=-\eta\left[\frac{m}{4\left(k p_{i}\right)} \gamma^{\mu} \hat{k} \hat{\varepsilon}_{ \pm}+\frac{m}{4\left(k p_{f}\right)} \hat{\varepsilon} \hat{\varepsilon} \hat{k} \gamma^{\mu}\right]
$$

and in case of circular polarization special functions $\mathrm{L}$ take the following form:

$$
L_{l}(\gamma, \chi)=e^{-i l \chi} J_{l}(\gamma)
$$


The $L$-functions have arguments of the following form:

$$
\gamma_{p p^{\prime}}=-\eta m \sqrt{-Q_{p p^{\prime}}^{2}}, \quad \operatorname{tg} \chi_{p p^{\prime}}=\frac{\left(Q_{p p^{\prime}} e_{y}\right)}{\left(Q_{p p^{\prime}} e_{x}\right)}
$$

where

$$
Q_{p p^{\prime}}=\frac{p}{(k p)}-\frac{p^{\prime}}{\left(k p^{\prime}\right)}
$$

In Equations (12) and (13) 4-momenta $p, p^{\prime}$ take values $\left(p_{i}, p_{f}\right)$ or $\left(p_{+}, p_{-}\right)$.

In Equation (8), index $l_{2}$ has a meaning of number of photons, absorbed in the second vertex. It should be noted that in weak fields processes with absorption of huge number of photons are strongly suppressed and starting from Equation (9) we are considering one photon absorption processes only $\left(l_{1}=l_{2}=1\right)$. Poles of the amplitude Equation (7) correspond to Oleinik resonances.

\section{Resonant Kinematics of Ultrarelativistic Electron-Positron Pair Production}

Under the resonance condition the process effectively reduces into two successive processes: X-ray-stimulated Compton effect and X-ray-stimulated Breit-Wheeler process (see Figure 2).

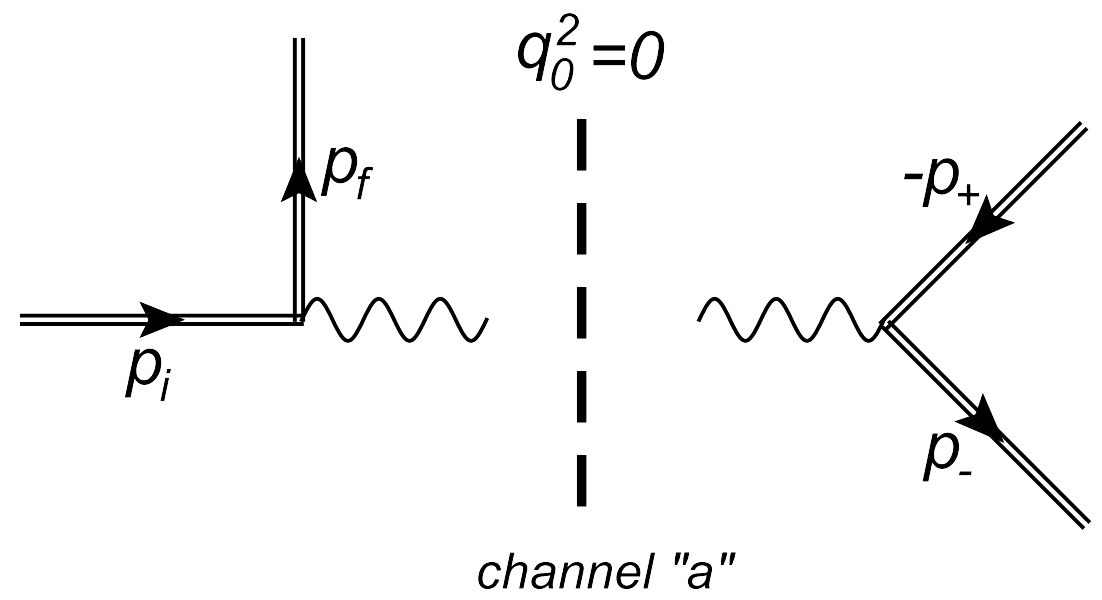

Figure 2. Feynman diagram of resonant electron-positron pair production by an electron in the field of an X-ray pulsar for channel "a". It is shown that under the resonance condition, the process is effectively reduced into two successive processes: X-ray-stimulated Compton effect and X-ray-stimulated Breit-Wheeler process.

It should be noted that channel " $\mathrm{b}$ " is obtained from channel "a" by change of indices $\left(p_{f} \longleftrightarrow p_{-}\right)$. Hence, it is sufficient to only consider kinematics of the channel "a".

Henceforth we denote 4-momentum of intermediate photon as $q_{0}$.

Let us consider the initial and final electrons and the particles of the electron-positron pair to be ultrarelativistic:

$$
E_{i, f} \gg m, \quad E_{ \pm} \gg m .
$$

In this case all the final particles (the two electrons and positron) propagate in a narrow cone along the direction of 3-momentum of the initial electron. The cone axis is far away from the direction of propagation of the $\mathrm{X}$-ray wave. If this condition is not satisfied, the Oleinik resonances are gone. According to this the angles subject to the following conditions:

$$
\theta_{i, f}=\angle\left(p_{i, f}, \boldsymbol{k}\right) \sim 1, \quad \theta_{ \pm}=\angle\left(\boldsymbol{p}_{ \pm}, \boldsymbol{k}\right) \sim 1
$$




$$
\theta_{f i}=\angle\left(\boldsymbol{p}_{f}, \boldsymbol{p}_{i}\right) \ll 1, \quad \theta_{ \pm i}=\angle\left(\boldsymbol{p}_{ \pm}, \boldsymbol{p}_{i}\right) \ll 1 .
$$

Starting from the 4-momentum conservation law in the first vertex

$$
p_{i}+k=p_{f}+q_{0}
$$

and using the conditions Equations (14)-(16) and the resonance condition

$$
q_{0}^{2}=0
$$

we get the equation for the energy of final electron:

$$
\left(1+4 \varepsilon_{i}+4 \varepsilon_{i}^{2} \delta_{f i}^{2}\right) x_{f}^{2}-2\left(1+2 \varepsilon_{i}\right) x_{f}+1=0
$$

Here

$$
x_{f}=\frac{E_{f}}{E_{i}}, \quad \varepsilon_{i}=\frac{E_{i}}{\omega_{t h r}}, \quad \delta_{f i}=\frac{\omega_{t h r} \theta_{f i}}{2 m}, \quad \omega_{t h r}=\frac{m^{2}}{\omega \sin ^{2} \frac{\theta_{i}}{2}} .
$$

In Equation (20) $\omega_{t h r}$ is the threshold energy for the intermediate photon (see Equation (33)).

From Equation (19) we obtain the resonant angle:

$$
\delta_{\text {res }}^{2}=\frac{-\left(1+4 \varepsilon_{i}\right) x_{f}^{2}+2\left(1+2 \varepsilon_{i}\right) x_{f}-1}{4 \varepsilon_{i}^{2} x_{f}^{2}},
$$

which is accomplished under strict resonance. In fact, there is zero probability to achieve strict resonance and real value of parameter $\delta_{f i}$ slightly differs from the one in Equation (21).

Solutions of Equation (19) give us dependence of the final electron energy on the parameter $\delta_{f i}$, proportional to its outgoing angle:

$$
x_{f}=\frac{1+2 \varepsilon_{i} \pm 2 \varepsilon_{i} \sqrt{1-\delta_{f i}^{2}}}{1+4 \varepsilon_{i}+4 \varepsilon_{i}^{2} \delta_{f i}^{2}}, \quad \delta_{f i}^{2} \leq 1 .
$$

From Equation (22), one can see that the parameter $\delta_{f i}$ takes values from closed interval $[0,1]$. Moreover, for each value of $\delta_{f i}$ the final electron's energy $x_{f}$ can take one of two possible values.

There, however, exists one particular. From consideration of 4-momentum conservation law in the second vertex one can find that X-ray-stimulated Breit-Wheeler process only goes if intermediate photon energy is greater than its threshold value $\omega_{t h r}$ (see Equation (20)), which is of order of $100 \mathrm{MeV}$ for x-radiation with $\omega \sim 1 \mathrm{KeV}$. This imposes a restriction on initial electron energy: it has to be greater than threshold energy $\varepsilon_{t h r}$, which exceeds the threshold energy of the intermediate photon:

$$
\varepsilon_{t h r}=\frac{1+\sqrt{2}}{2}
$$

and also a restriction on final electron energy:

$$
x_{f} \leq 1-\frac{1}{\varepsilon_{i}} .
$$

With the presence of restriction Equations (24) there appears one more characteristic value of energy of the initial electron:

$$
\varepsilon_{*}=\frac{1+\sqrt{3}}{2}
$$


which has the next meaning (see Figure $3 \mathrm{a}, \varepsilon_{i}=\varepsilon_{*}$ ): as long as initial electron energy is less than $\varepsilon_{*}$ final electron energy can only take one (low) value, otherwise, for large enough values of parameter $\delta_{f i}$, the final electron energy can take one of two possible values.

Subject to this comment, final electron energy dependence on $\delta_{f i}$ takes the form as shown in Figure 3.
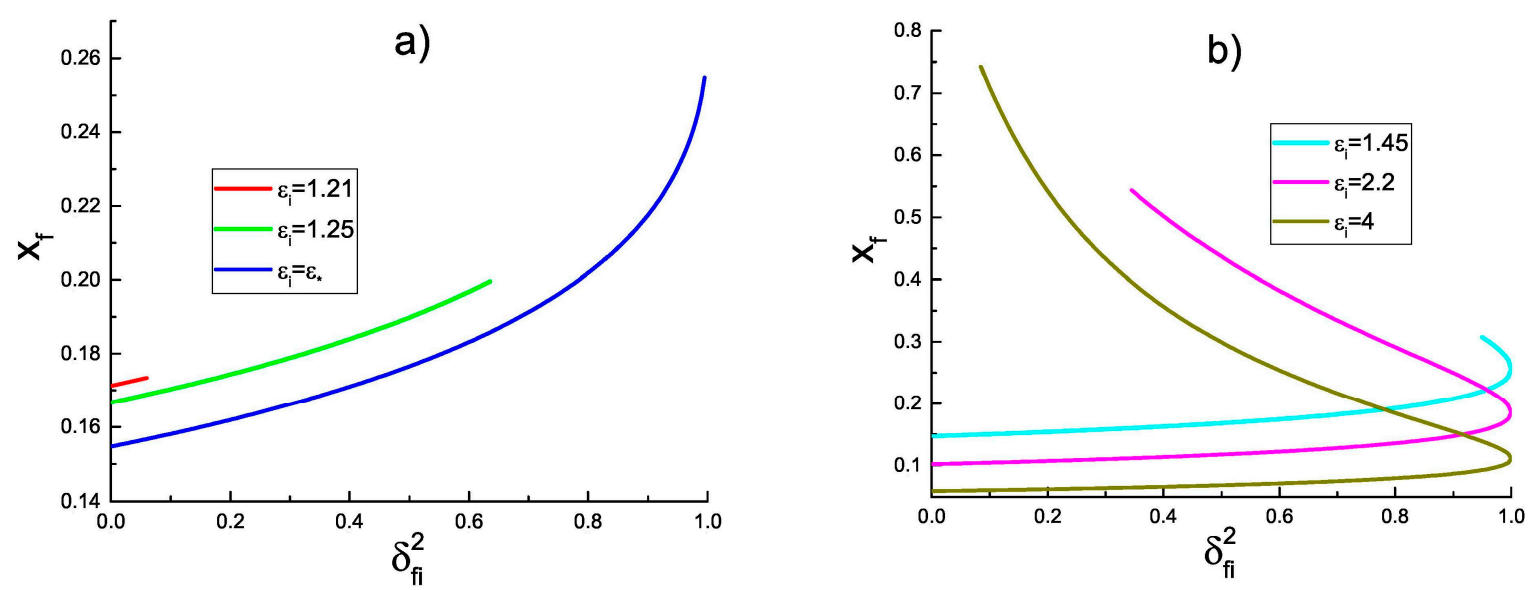

Figure 3. (a) The energy of the final electron as a function of parameter $\delta_{f i}^{2}$ for different values of energy of the initial electron from the closed interval $\varepsilon_{i} \in\left[\varepsilon_{t h r}, \varepsilon_{*}\right]$. (b) The energy of final electron as a function of parameter $\delta_{f i}^{2}$ for different values of energy of the initial electron satisfying the condition $\varepsilon_{i}>\varepsilon_{*}$.

Let us now show how the threshold energy $\omega_{t h r}$ Equation (20) for X-ray-stimulated Breit-Wheeler process appears.

Starting from 4-momentum conservation law in the second vertex:

$$
q_{0}+k=p_{-}+p_{+}
$$

and using the conditions Equations (14)-(16) and (18) we get the equation for the energy of the positron:

$$
\left(\varepsilon_{i}+\varepsilon_{i}^{2} \delta_{0+}^{2} x_{0}\right) x_{+}^{2}-\varepsilon_{i} x_{0} x_{+}+\frac{x_{0}}{4}=0,
$$

where

$$
\begin{gathered}
x_{0}=\frac{E_{0}}{E_{i}}, \quad x_{+}=\frac{E_{+}}{E_{i}}, \\
\delta_{0+}=\frac{\omega_{t h r} \theta_{0+}}{2 m}, \quad \theta_{0+}=\angle\left(\boldsymbol{p}_{0}, \boldsymbol{p}_{+}\right) \ll 1
\end{gathered}
$$

and $p_{0}=\left(E_{0}, \boldsymbol{p}_{0}\right)$ is the 4-momentum of the intermediate photon.

The discriminant of Equation (27) is

$$
D_{0}=\varepsilon_{i}^{2} x_{0}^{2}-x_{0}\left(\varepsilon_{i}+\varepsilon_{i}^{2} \delta_{0+}^{2} x_{0}\right) .
$$

The condition of existence of solutions of Equation (27) is the following:

$$
D_{0} \geq 0 \text {, }
$$

which leads to

$$
x_{0} \geq \frac{1}{\varepsilon_{i}\left(1-\delta_{0+}^{2}\right)} .
$$


Thus, with definitions of $\varepsilon_{i}$ Equation (20) and $x_{0}$ Equation (28), the minimal value which intermediate photon energy can take is

$$
E_{0}=\omega_{t h r}
$$

Let us now consider kinematics of electron and positron of pair. We, again, start from 4-momentum conservation law in the second vertex Equation (26).

From Equation (26) using the conditions Equations (14)-(16) and (18) one can get the equation for the energy of the positron of pair.

$$
\left(1+4 \varepsilon_{i}^{2} \delta_{+i}^{2}-\frac{1}{x_{f}}-4 \varepsilon_{i}^{2} \delta_{f+}^{2} x_{f}+8 \varepsilon_{i}\right) x_{+}^{2}-4 \varepsilon_{i}\left(1-x_{f}\right) x_{+}+\left(1-x_{f}\right)=0 .
$$

Here

$$
\begin{gathered}
\theta_{f+}=L\left(\boldsymbol{p}_{f}, \boldsymbol{p}_{+}\right) \ll 1, \\
\delta_{+i}^{2}=\frac{\omega_{t h r}^{2} \theta_{+i}^{2}}{4 m^{2}}, \quad \delta_{f+}^{2}=\frac{\omega_{t h r}^{2} \theta_{f+}^{2}}{4 m^{2}}
\end{gathered}
$$

and parameter $\delta_{f+}^{2}$ should be treated as follows:

$$
\delta_{f+}^{2}=\delta_{f i}^{2}+\delta_{+i}^{2}-2 \delta_{f i} \delta_{+i} \cos \left(\varphi_{f i}-\varphi_{+i}\right) .
$$

In Equation (34) $x_{f}$ is not an independent variable but it stands for the right-hand side of the Equation (22).

The discriminant of Equation (34) is

$$
D=16 \varepsilon_{i}^{2}\left(1-x_{f}\right)^{2}-4\left(1-x_{f}\right)\left(1+4 \varepsilon_{i}^{2} \delta_{+i}^{2}-\frac{1}{x_{f}}-4 \varepsilon_{i}^{2} \delta_{f+}^{2} x_{f}+8 \varepsilon_{i}\right) .
$$

The solutions of Equation (34) are

$$
x_{+}=\frac{4 \varepsilon_{i}\left(1-x_{f}\right) \pm \sqrt{D}}{2\left(1+4 \varepsilon_{i}^{2} \delta_{+i}^{2}-\frac{1}{x_{f}}-4 \varepsilon_{i}^{2} \delta_{f+}^{2} x_{f}+8 \varepsilon_{i}\right)} .
$$

From energy conservation law

$$
x_{-} \approx 1-x_{f}-x_{+}
$$

one can obtain the energy of the electron of the pair:

$$
x_{-}=1-x_{f}-\frac{4 \varepsilon_{i}\left(1-x_{f}\right) \pm \sqrt{D}}{2\left(1+4 \varepsilon_{i}^{2} \delta_{+i}^{2}-\frac{1}{x_{f}}-4 \varepsilon_{i}^{2} \delta_{f+}^{2} x_{f}+8 \varepsilon_{i}\right)} .
$$

In spite it seeming there are only two possible values for the energies of the particle pair, there are actually four: two for each value of the final electron energy $x_{f}$ (see Figure $4 \mathrm{~b}, \mathrm{c}$ ). However, for small values of initial electron energy or for small values of parameter $\delta_{f i}$ only one possible value of $x_{f}$ is available which leads to only two possible values of pair particles energies (see Figure 4a).

From Figure $4 \mathrm{a}-\mathrm{c}$ we can sequentially follow the evolution of dependencies of the positron Equation (39) and the electron Equation (41) energies as functions of parameter $\delta_{+i}$ as initial electron energy increases from $\varepsilon_{i}=\varepsilon_{*}$ in Figure 4 a to $\varepsilon_{i}=4$ in Figure $4 \mathrm{c}$. When the initial electron energy is low (Figure 4a) particles of the pair cannot propagate at either large or small angles, i.e., there exist closed interval $\left[\delta_{+i}^{\min }, \delta_{+i}^{\max }\right]$, where $\delta_{+i}^{\min } \neq 0$. Moreover, in this case only two possible values of energies of the particles are available for each angle $\delta_{+i}$. In Figure $4 \mathrm{~b}$, with a little increase in initial electron 
energy, there appears a possibility for the particles of the pair to propagate along the direction of initial electron 3-momentum $\left(\delta_{+i}=0\right)$ and also there all the four possible values of energies are available for some values of $\delta_{+i}$, whose appearance is linked with the existence of a second (high) value of the final electron energy $x_{f}$. In Figure $4 c$ the dependencies considered take the form similar to what we have seen in Figure $3 \mathrm{a}, \mathrm{b}$ for the final electron energy $x_{f}$.
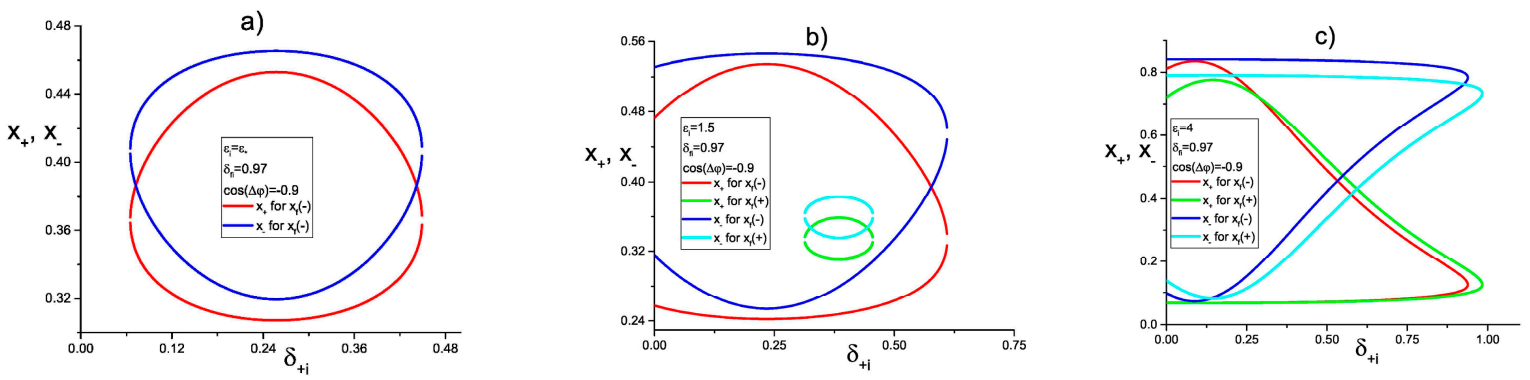

Figure 4. (a) Energies of the positron (red) and the electron (blue) of pair as functions of parameter $\delta_{+i}$ for initial electron energy $\varepsilon_{i}=\varepsilon_{*}$, parameter $\delta_{f i}=0.97$, the final electron energy $x_{f}(0.97) \approx 0.23$ and azimuthal angles difference $\cos \left(\varphi_{f i}-\varphi_{+i}\right)=-0.9$. (b) The positron (red) and the electron (blue) energies as functions of parameter $\delta_{+i}$ for low final electron energy $x_{f}(0.97 ;-) \approx 0.21$ and the positron (green) and the electron (cyan) energies as functions of parameter $\delta_{+i}$ for high final electron energy $x_{f}(0.97 ;+) \approx 0.31$ for initial electron energy $\varepsilon_{i}=1.5$, parameter $\delta_{f i}=0.97$, and azimuthal angle difference $\cos \left(\varphi_{f i}-\varphi_{+i}\right)=-0.9$. (c) The positron (red) and the electron (blue) energies as functions of parameter $\delta_{+i}$ for low final electron energy $x_{f}(0.97 ;-) \approx 0.09$ and the positron (green) and electron (cyan) energies as functions of parameter $\delta_{+i}$ for high final electron energy $x_{f}(0.97 ;+) \approx 0.14$ for initial electron energy $\varepsilon_{i}=4$, parameter $\delta_{f i}=0.97$, and azimuthal angles difference $\cos \left(\varphi_{f i}-\varphi_{+i}\right)=-0.9$.

Analysis of discriminant Equation (38) gives us a surface in $\left(\varepsilon_{i}, \delta_{f i}, \cos \left(\varphi_{f i}-\varphi_{+i}\right)\right)$-parametric space which separates the region in this space where the process is kinematically allowed from the region where the process in kinematically forbidden. The regions are shown in Figure 5a for high value of the final electron energy and Figure $5 b$ for the low one. From Equation (38) it also could be obtained that the parameter $\delta_{+i}$ takes values from the closed interval $[0,1]$ for low values of the final electron energy and from the closed interval $[0, \sqrt{2}]$ for high values of the final electron energy.
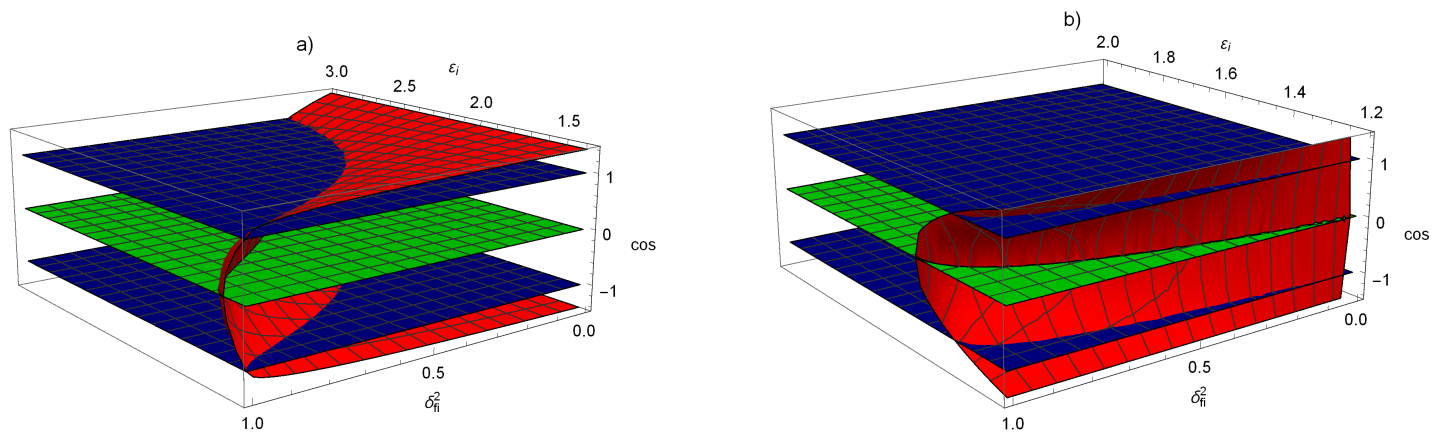

Figure 5. Surface (red) in $\left(\varepsilon_{i}, \delta_{f i}, \cos \left(\varphi_{f i}-\varphi_{+i}\right)\right)$-parametric space which separates the region in this space where the process is kinematically allowed from the region where the process in kinematically forbidden for high (a) and low (b) values of the final electron energy. Blue planes correspond to $\cos \left(\varphi_{f i}-\varphi_{+i}\right)= \pm 1$ and the green one to $\cos \left(\varphi_{f i}-\varphi_{+i}\right)=0$. 


\section{Resonant Kinematics of Ultrarelativistic Electron-Positron Pair Production by High-Energy} Electrons in the Field of an X-ray Pulsar for High Initial Electron Energy

Let us consider the case of 100-GeV-energy initial electrons, which are required to obtain 100-GeV-energy positrons discussed in [1]. This corresponds to the condition

$$
\varepsilon_{i}=1000 \gg 1 \text {. }
$$

According to Equation (42) final electron energy Equation (22) simplifies to

$$
x_{f}=\frac{1}{2} \frac{1 \pm \sqrt{1-\delta_{f i}^{2}}}{1+\varepsilon_{i} \delta_{f i}^{2}}
$$

and the positron energy Equation (39) simplifies to

$$
x_{+}=\frac{1}{1+\varepsilon_{i} \delta_{+i}^{2}}
$$

for both low and high final electron energies and two more values of the positron energy are vary low, so we are not considering them. The energy of the electron in the pair could be obtained with the use of the energy conservation law Equation (40).

From Figure 6 we can see that the energy of the positron of pair takes its maximal value, which is of order of the energy of the initial electron, when the positron propagates along the initial electron 3-momentum direction.

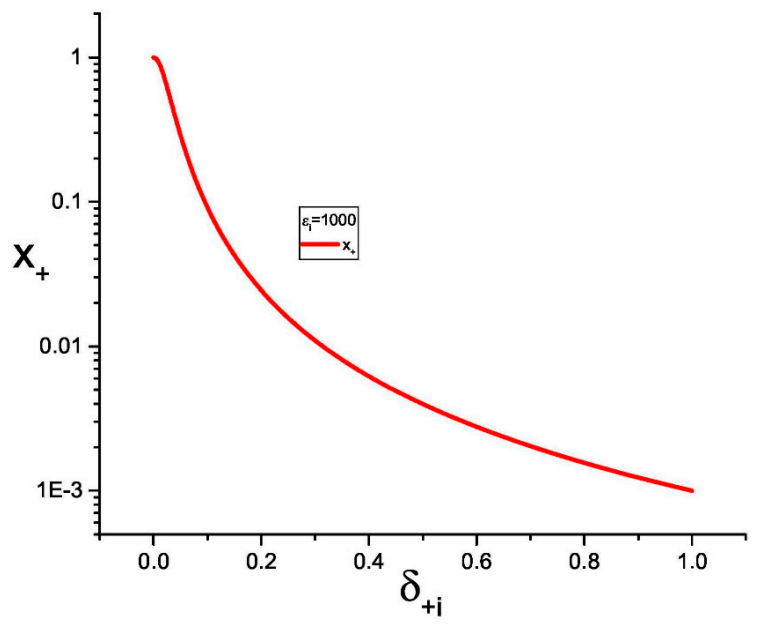

Figure 6. Positron energy as function of parameter $\delta_{+i}$ for initial electron energy $\varepsilon_{i}=1000$.

\section{Differential Probability (Per Unit Time) of Resonant Ultrarelativistic Electron-Positron Pair} Production by High-Energy Electrons in the Field of an X-ray Pulsar

Differential probability (per unit time) of the process is defined as follows:

$$
d w=\frac{1}{(2 \pi)^{9}}|S|^{2} d^{3} p_{f} d^{3} p_{-} d^{3} p_{+}
$$

where the amplitude $S$ is defined by Equation (7).

Henceforth we will only consider differential probability for channel " $a$ " as far as the one for the channel " $\mathrm{b}$ " can be obtained by change of indices $\left(p_{f} \longleftrightarrow p_{-}\right)$and we are not interested in interference terms in this paper. 
The differential probability for channel "a" after summing and averaging over the final and initial state particles' polarizations, respectively, takes the following form:

$$
d w=\frac{\alpha^{2} \eta^{4}}{32 \pi^{3}} \frac{E_{i}^{3}}{E_{f} E_{-} E_{+}} G \frac{V_{C} U_{B W}}{\left|\left(p_{i}-p_{f}+k\right)^{2}\right|^{2}} \delta^{(4)}\left(p_{i}-p_{f}-p_{-}-p_{+}+2 k\right) d^{3} p_{f} d^{3} p_{-} d^{3} p_{+} .
$$

In Equation (46) $G$ is some function of 4-momenta, whose magnitude is of order of $\sim 1, V_{C}$ is the function defining the probability (per unit time) of X-ray-stimulated Compton effect and $U_{B W}$ is the function defining the probability (per unit time) of X-ray-stimulated Breit-Wheeler process. These functions are of order of $\sim 1$ and of the following form:

$$
\begin{gathered}
V_{C}=\frac{1}{(1+v)^{2}}\left[2+\frac{v^{2}}{1+v}-\frac{4 v}{v_{1}}\left(1-\frac{v}{v_{1}}\right)\right], \\
U_{B W}=\frac{1}{u \sqrt{u(u-1)}}\left[2 u-1+\frac{2 u}{u_{1}}\left(1-\frac{u}{u_{1}}\right)\right]
\end{gathered}
$$

where $v, v_{1}, u, u_{1}$ are kinematic invariants:

$$
\begin{array}{cc}
u=\frac{\left(\left(k p_{+}\right)+\left(k p_{-}\right)\right)^{2}}{4\left(k p_{+}\right)\left(k p_{-}\right)}=\frac{\left(x_{-}+x_{+}\right)^{2}}{4 x_{-} x_{+}}, & u_{1}=\frac{\left(k p_{+}\right)+\left(k p_{-}\right)}{2 m^{2}}=\varepsilon_{i}\left(x_{-}+x_{+}\right) \\
v=\frac{\left(k p_{i}\right)+\left(k p_{f}\right)}{\left(k p_{f}\right)}=\frac{1+x_{f}}{x_{f}}, & v_{1}=\frac{2\left(k p_{i}\right)}{m^{2}}=4 \varepsilon_{i} .
\end{array}
$$

Under the resonance condition the denominator in Equation (46) turns to zero. To eliminate this resonance infinity the Breit-Wigner procedure is applied-an imaginary term, corresponding to finite lifetime of initial electron, should be added to its energy:

$$
E_{i} \longrightarrow E_{i}^{\prime}=E_{i}-i \Gamma
$$

After applying substitution Equation (51) the denominator turns to

$$
\left|\left(p_{i}-p_{f}+k\right)^{2}\right|^{2}=4 E_{i}^{2}\left(1-x_{f}\right)^{2}\left(\frac{4 m^{4} \varepsilon_{i}^{4} x_{f}^{2}}{E_{i}^{2}\left(1-x_{f}\right)^{2}}\left[\delta_{f i}^{2}-\delta_{r e s}^{2}\right]^{2}+\Gamma^{2}\right)
$$

where $\Gamma=\frac{1}{2} W_{C}$ and $W_{C}$ is the total probability (per unit time) of X-ray-stimulated Compton effect. $W_{C}$ is given [30] by the following expression:

$$
W_{C}=\frac{e^{2} m^{2}}{32 \pi E_{i}} \eta^{2} W^{\prime}
$$

where

$$
W^{\prime}=\left[\left(1-\frac{4}{v_{1}}-\frac{8}{v_{1}^{2}}\right) \ln \left(1+v_{1}\right)+\frac{1}{2}+\frac{8}{v_{1}}-\frac{1}{2\left(1+v_{1}\right)^{2}}\right] .
$$

Finally, after the integration with $\delta$-function, the differential probability (per unit time) of resonant electron-positron pair production by an electron in the field of an X-ray pulsar is given as follows:

$$
d w_{a}=\frac{\alpha^{2} \eta^{4}}{32 \pi^{3}} \frac{m^{4} E_{i}^{3}}{\omega_{t h r}^{4}} \frac{x_{f} x_{-}}{x_{+}\left(1-x_{f}\right)^{2}} G \frac{V_{C} U_{B W}}{B_{i f}^{2}\left[\delta_{f i}^{2}-\delta_{r e s}^{2}\right]^{2}+\Gamma^{2}} d x_{f} d \delta_{f i}^{2} d \delta_{+i}^{2} d \varphi_{f i} d \varphi_{+i},
$$


where

$$
B_{i f}=\frac{2 m^{2} \varepsilon_{i}^{2} x_{f}}{E_{i}\left(1-x_{f}\right)} .
$$

Equation (55) reaches its maximum value under the resonance condition when $\delta_{f i} \rightarrow \delta_{\text {res }}$. In this case it takes the following form:

$$
d w_{a}^{\max }=\frac{128}{\pi \alpha^{2} \eta^{4}}\left[\alpha^{2} \eta^{4} \frac{E_{i}^{5}}{\omega_{t h r}^{4}} \frac{x_{f} x_{-}}{x_{+}\left(1-x_{f}\right)^{2}} G \frac{V_{C} U_{B W}}{W^{\prime 2}}\right] d x_{f} d \delta_{f i}^{2} d \delta_{+i}^{2} d \varphi_{f i} d \varphi_{+i} .
$$

Away from resonance, when $\left|\delta_{f i}^{2}-\delta_{r e s}^{2}\right| \gg \frac{\Gamma}{B_{i f}}$, expression in square brackets in Equation (57) defines the order of differential probability (per unit time) of the non-resonant process, which is proportional to $\alpha^{2}$ as far as it is the process of the second order in fine structure constant and to $\eta^{4}$ for each of fermion lines.

Denoting the differential probability (per unit time) of the non-resonant process as $d w_{a}^{\text {nonres }}$ from Equation (57) we can write

$$
d w_{a}^{\max } \sim \frac{128}{\pi \alpha^{2} \eta^{4}} d w_{a}^{\text {nonres }} .
$$

From Equation (58) we can estimate resonant differential probability (per unit time) compared with the non-resonant one. The result of this estimation is that the resonant differential probability exceeds the non-resonant one by 14 orders of magnitude for $\eta=0.01$, which corresponds to $\omega=1$ $\mathrm{KeV}$ and $F=10^{11} \mathrm{~V} / \mathrm{cm}$.

It should be underlined that the differential probability (per unit time) estimate we provide corresponds to its maximal value. In fact, the real X-ray pulsar field is inhomogeneous in space and time which leads to resonance width to be much greater than radiation width. Due to this, the value of resonance differential probability (per unit time) of the process becomes several orders of magnitude smaller.

\section{Conclusions}

The process of resonant ultrarelativistic electron-positron pair production by high-energy electrons in the field of an X-ray pulsar has several features. All the final particles propagate in a narrow cone along the direction of the initial electron 3-momentum. Because of the X-ray-stimulated Breit-Wheeler process, which occurs in the second vertex, having threshold energy the whole process considered has threshold energy Equation (23). For x-radiation of pulsars with $\omega=1 \mathrm{KeV}$ the threshold energy is of order of $100 \mathrm{MeV}$, which is three orders less than the same value for optical lasers with radiation with frequencies of order of $1 \mathrm{eV}$. This fact makes it easier for the discussed process to occur in the presence of pulsar radiation rather than in the field of optical lasers.

The thing that dramatically distinguishes the resonant process from the non-resonant one is the presence of Oleinik resonances, whose existence is connected with a discrete spectrum of particle + external field system. Due to them, there exists strong dependence of energies of final particles on their outgoing angles (see Equations (22), (39) and (41)). As shown in Figures 3 and 4 there are several possible values of energies of all the final particles for each value of their outgoing angles and up to four values of pair particle energies for certain outgoing angles (see Figure $4 b, c)$.

We analyzed the conditions under which the process is kinematically allowed. Corresponding regions are shown in Figure 5a,b. Restrictions on outgoing angles were also found.

To explain anomalous positron abundance in the 100-GeV region reported in [1] high energy initial electrons were considered. It is shown that such positrons may occur at low outgoing angles (see Figure 6).

Under the resonance condition, an intermediate photon becomes a real particle which leads to resonance infinity in the differential probability. To eliminate this infinity, the Breit-Wigner procedure 
is applied. The differential probability (per unit time) of resonant process is estimated. It exceeds the differential probability (per unit time) of the non-resonant process by 14 orders of magnitude for parameter $\eta=0.01$, which can be obtained near pulsars with $\omega=1 \mathrm{KeV}$ and $F=10^{11} \mathrm{~V} / \mathrm{cm}$.

Author Contributions: Conceptualization, S.P.R.; methodology, S.P.R. and V.V.D.; software, G.K.S.; validation, S.P.R., V.V.D. and G.K.S.; formal analysis, S.P.R. and G.K.S.; investigation, S.P.R., V.V.D. and G.K.S.; resources, V.V.D.; data curation, S.P.R. and V.V.D.; writing — original draft preparation, G.K.S.; writing — review and editing, S.P.R. and G.K.S.; visualization, G.K.S.; supervision, S.P.R. and V.V.D.; project administration, V.V.D.; funding acquisition, V.V.D. All authors have read and agreed to the published version of the manuscript.

Funding: This research received no external funding.

Acknowledgments: Georgii Sizykh acknowledges helpful discussions with Nikita R. Larin and Dmitriy V. Doroshenko.

Conflicts of Interest: The authors declare no conflict of interest.

\section{References}

1. Hooper, D.; Blasi, P.; Serpico, P.D. Pulsars as the sources of high energy cosmic ray positrons. J. Cosmol. Astropart. Phys. 2009, 2009, 025. [CrossRef]

2. Adriani, O.; Barbarino, G.C.; Bazilevskaya, G.A.; Bellotti, R.; Boezio, M.; Bogomolov, E.A.; Bonechi, L.; Bongi, M.; Bonvicini, V.; Bottai, S.; et al. An anomalous positron abundance in cosmic rays with energies 1.5-100 GeV. Nature 2009, 458, 607-609. [CrossRef] [PubMed]

3. Oleinik, V. Resonance effects in the field of an intense laser beam. J. Exp. Theor. Phys. 1967, 25, 697.

4. Oleinik, V. Resonance effects in the field of an intense laser ray. J. Exp. Theor. Phys. 1968, 26, 1132.

5. Roshchupkin, S.P. Resonant effects in collisions of relativistic electrons in the field of a light wave. Laser Phys. 1996, 6, 837-858.

6. Sergei, R.; Lebed', A.; Elena, P.; Voroshilo, A. Quantum electrodynamics resonances in a pulsed laser field. Laser Phys. 2012, 22, 1113.

7. Dubov, A.; Dubov, V.V.; Roshchupkin, S.P. Resonant high-energy bremsstrahlung of ultrarelativistic electrons in the field of a nucleus and a weak electromagnetic wave. Laser Phys. Lett. 2020, 17, 045301. [CrossRef]

8. Roshchupkin, S. Resonant electron-electron scattering in the field of a light wave: The general relativistic case. Laser Phys. 1994, 4, 221-224.

9. Voroshilo, O.; Roshchupkin, S. Resonant two-photon emission of an electron in the field of an electromagnetic wave. In Problems of Atomic Science and Technology; National Science Center "Kharkiv Institute of Physics and Technology": Kharkiv, Ukraine, 2007.

10. Roshchupkin, S.P.; Lebed', A.A. Effects of Quantum Electrodynamics in the Strong Pulsed Laser Fields; Naukova Dumka: Kiev, Ukraine, 2013.

11. Roshchupkin, S.P. Resonant spontaneous bremsstrahlung of an electron in the field of the nucleus and two light waves. Laser Phys. 2002, 12, 498-503.

12. Voroshilo, A.I.; Roshchupkin, S.P. Resonant scattering of a photon by an electron in the field of a circularly polarized electromagnetic wave. Laser Phys. Lett. 2005, 2, 184-189. [CrossRef]

13. Voroshilo, A.; Roshchupkin, S.; Denisenko, O. Resonance of exchange amplitude of Compton effect in the circularly polarized laser field. Eur. Phys. J. D 2007, 41, 433-440. [CrossRef]

14. Lebed', A.A.; Roshchupkin, S.P. Resonant spontaneous bremsstrahlung by an electron scattered by a nucleus in the field of a pulsed light wave. Phys. Rev. A 2010, 81, 033413. [CrossRef]

15. Nedoreshta, V.; Voroshilo, A.; Roshchupkin, S. Resonant scattering of an electron by a muon in the field of light wave. Eur. Phys. J. D 2008, 48, 451-458. [CrossRef]

16. Larin, N.R.; Dubov, V.V.; Roshchupkin, S.P. Resonant production of electron-positron pairs by a hard gamma-ray on a nucleus in an external electromagnetic field. Mod. Phys. Lett. A 2020, 35, 2040025. [CrossRef]

17. Dubov, A.; Dubov, V.; Roshchupkin, S. Resonant emission of hard gamma-quanta at scattering of ultrarelativistic electrons on a nucleus within the external light field. Mod. Phys. Lett. A 2020, 35, 2040024. [CrossRef]

18. Doroshenko, D.; Dubov, V.; Roshchupkin, S. Resonant annihilation and production of high-energy electron-positron pairs in an external electromagnetic field. Mod. Phys. Lett. A 2020, 35, 2040023. [CrossRef] 
19. Nedoreshta, V.N.; Roshchupkin, S.P.; Voroshilo, A.I. Resonance of the exchange amplitude of a photon by an electron scattering in a pulsed laser field. Phys. Rev. A 2015, 91, 062110. [CrossRef]

20. Nedoreshta, V.N.; Voroshilo, A.I.; Roshchupkin, S.P. Resonant scattering of a photon by an electron in the moderately-strong-pulsed laser field. Phys. Rev. A 2015, 88, 052109. [CrossRef]

21. Lebed', A.A.; Roshchupkin, S.P. Nonresonant photocreation of electron-positron pair on a nucleus in the field of a pulsed light wave. Laser Phys. 2011, 21, 1613. [CrossRef]

22. Larin, N.R.; Dubov, V.V.; Roshchupkin, S.P. Resonant photoproduction of high-energy electron-positron pairs in the field of a nucleus and a weak electromagnetic wave. Phys. Rev. A 2019, 100, 052502. [CrossRef]

23. Mackenroth, F.; Di Piazza, A. Nonlinear trident pair production in an arbitrary plane wave: A focus on the properties of the transition amplitude. Phys. Rev. D 2018, 98, 116002. [CrossRef]

24. Dinu, V.; Torgrimsson, G. Trident pair production in plane waves: Coherence, exchange, and spacetime inhomogeneity. Phys. Rev. D 2018, 97, 036021. [CrossRef]

25. Hu, H.; Müller, C.; Keitel, C.H. Complete QED theory of multiphoton trident pair production in strong laser fields. Phys. Rev. Lett. 2010, 105, 080401. [CrossRef] [PubMed]

26. Ilderton, A. Trident pair production in strong laser pulses. Phys. Rev. Lett. 2011, 106, 020404. [CrossRef] [PubMed]

27. King, B.; Ruhl, H. Trident pair production in a constant crossed field. Phys. Rev. D 2013, 88. [CrossRef]

28. Volkov, D.M. Über eine klasse von lösungen der diracschen gleichung. Z. Phys. 1935, 94, 250-260. [CrossRef]

29. Berestetskii, V.B.; Pitaevskii, L.P.; Lifshitz, E.M. Quantum Electrodynamics; Butterworth-Heinemann: Oxford, UK, 1982.

30. Ritus, V.I.; Nikishov, A.I. Quantum electrodynamics phenomena in the intense field. In Trudy FIAN; Nauka: Moscow, Russia, 1979; Volume 111.

(C) 2020 by the authors. Licensee MDPI, Basel, Switzerland. This article is an open access article distributed under the terms and conditions of the Creative Commons Attribution (CC BY) license (http://creativecommons.org/licenses/by/4.0/). 